\title{
Parametric studies of ring and parallel coupled line resonators for matched bandstop filter design
}

\author{
Abdullah M. Zobilah ${ }^{1}$, Adib Othman², Noor Azwan Shairi ${ }^{3}$, Zahriladha Zakaria $^{4}$ \\ 1,2,3,4 Microwave Research Group (MRG), Centre for Telecommunication Research \& Innovation (CeTRI), \\ Universiti Teknikal Malaysia Melaka (UTeM), Malaysia \\ ${ }^{1,3,4}$ Fakulti Kejuruteraan Elektronik dan Kejuruteraan Komputer (FKEKK), Universiti Teknikal Malaysia Melaka \\ (UTeM), Malaysia \\ ${ }^{2}$ Fakulti Teknologi Kejuruteraan Elektrik dan Elektronik (FTKEE), Universiti Teknikal Malaysia Melaka (UTeM), \\ Malaysia
}

\begin{abstract}
Article Info
Article history:

Received Jun 1, 2018

Revised Nov 10, 2018

Accepted Jan 7, 2019

Keywords:
Dual mode
Matched bandstop filter
Parallel coupled resonator
Ring resonator

Keywords:

ual mode

Ring resonator

ABSTRACT

Recently, matched bandstop filters had become substantial part of modern RF and microwave systems. However, in these types of filters, the key problem in the couplings of any microstrip resonators to transmission microstrip line is the variation or tolerance of coupling gap. It was found that the bandstop response is very sensitive to the gap size of the coupled line. Therefore, this paper presents parametric studies of dual mode parallel coupled line and ring resonator for matched bandstop filter design. For parallel coupled line resonator, it was found that with careful design and proper circuit parametric study on the coupling spacing, very high notch and matched return loss response were obtained. In contrast, for ring resonator, based on the simulated result, it was found that a very high notch and matched return loss response were obtained with careful design and proper circuit parametric study on the coupling spacing, width at coupling lines, and perturbed stub length.
\end{abstract}

Copyright (C) 2019 Institute of Advanced Engineering and Science. All rights reserved.

\section{Corresponding Author:}

\section{N. A. Shairi,}

Microwave Research Group (MRG),

Centre for Telecommunication Research \& Innovation (CeTRI),

Fakulti Kejuruteraan Elektronik dan Kejuruteraan Komputer (FKEKK),

Universiti Teknikal Malaysia Melaka (UTeM),

Hang Tuah Jaya, 76100, Durian Tunggal, Melaka, Malaysia

Email: noorazwan@utem.edu.my

\section{INTRODUCTION}

In general, bandstop filters (BSF) are commonly used for rejecting unwanted blocking and interference signals in RF and microwave systems [1], [2]. It is well known that the planar technologies, particularly those using microstrip, suffer from low Q factor compared to non-planar technologies, such as coaxial and rectangular waveguides [3]. High notch depth and selectivity of bandstop filters are usually difficult to achieve with low $\mathrm{Q}$ factor of lossy resonator, unless multiple lossy resonators are placed in the design for higher n-order of bandstop filter. However, it tends to be physically large and complex.

A study issued in 2005 could successfully demonstrate an ideal infinite stopband attenuation of the matched bandstop, where high notch depth and selectivity can be produced with only two lossy low-Q resonators in microstrip technology [4]. Figure 1 presents the generalized model of matched bandstop filter using lossy resonator.

After the first prototypes from [4], other distributed elements of lossy resonators or different shapes (such as ring or coupled line) of matched bandstop filters have been proposed and reported by other researchers [5]-[12]. Besides, the matched bandstop filter was also realized by using lumped element type of resonator [13], [14]. 
Recently, modern RF and microwave systems started tending towards cognitive operations thus, more tunable and switchable filters were conducted. For instance, a matched bandstop filter with the feature of switching between bandstop and all-pass was reported using ring resonator [15] and parallel-coupled resonator [16]. Furthermore, there were switchable matched bandstop to band-pass filter designs utilizing ring resonator [17] and parallel-coupled resonator [18]. Besides, the potential application of tunable matched bandstop filter in SPDT switch was discussed in [19]-[23].

In these matched bandstop filter designs, the key problem is the couplings of any microstrip resonators to the transmission microstrip line that have the variation or tolerance of coupling gap. As reported in [15], it was found that the bandstop response is very sensitive to the gap size of the coupled line. The smallest changes of coupling gap could significantly change the response. Unfortunately, the previous reported studies did not analyze and discusses the designing process of matched bandstop filter using lossy resonators. Therefore, in this paper, parametric studies of dual mode parallel coupled line and ring resonator for matched bandstop filter design are analyzed and discussed for helping and guiding other researchers who intend to deal with these type of matched bandstop filters.

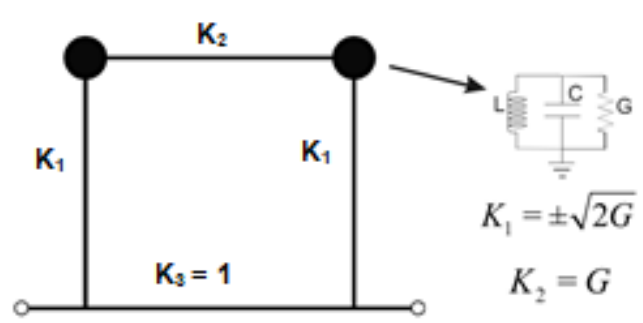

Figure 1. Generalized model of matched bandstop filter [4]

\section{THEORETICAL OF MATCHED BANDSTOP FILTER}

This is a simple analysis and discussion of attenuation response of matched lossy resonators. The theory of matched bandstop filter using lossy resonator was derived mathematically from mathematical model of allpass network [4]. Consider an even-mode and odd-mode analyses of matched bandstop filter, a transfer matrix of the symmetrical network is given by

$$
\left[T_{R}\right]=\left[\begin{array}{cc}
\frac{Y_{e}+Y_{o}}{Y_{o}-Y_{e}} & \frac{2}{Y_{o}-Y_{e}} \\
\frac{2 Y_{e} Y_{o}}{Y_{o}-Y_{e}} & \frac{Y_{e}+Y_{o}}{Y_{o}-Y_{e}}
\end{array}\right]
$$

where Ye is even-mode, and Yo is odd-mode of admittance of the resonator. If $Y_{o}=\frac{1}{Y_{e}}$, the general mathematical model of $S_{21}$ of matched lossy resonator was rewritten as

$$
S_{21}=\frac{\left(\frac{1}{Y_{e}}-Y_{e}\right) Z_{0}}{\left(\frac{Z_{0}}{Y_{e}}+1\right)\left(Z_{0} Y_{e}+1\right)}=\frac{\left(1-Y_{e}^{2}\right) Z_{0}}{\left(Z_{0}+Y_{e}\right)\left(Z_{0} Y_{e}+1\right)}
$$

Now consider the case where $Y_{e}$ is a lossy circuit, $Y_{e}=R+j \omega L+\frac{1}{j \omega C}$. Then, (2) was rewritten as

$$
S_{21}=\frac{\left(1-\left(R+j \omega L-\frac{j}{\omega C}\right)^{2}\right) Z_{0}}{\left(Z_{0}+\left(R+j \omega L-\frac{j}{\omega C}\right)\right)\left(Z_{0}\left(R+j \omega L-\frac{j}{\omega C}\right)+1\right)}
$$


From (3), the resonant results when

$$
j \omega L-\frac{j}{\omega C}=0
$$

Rearrange (3) with (4), then we get

$$
S_{21}=\frac{\left(1-R^{2}\right) Z_{0}}{\left(Z_{0}+R\right)\left(Z_{0} R+1\right)}
$$

Consider a normalized characteristic impedance where $\mathrm{Z}_{\mathrm{O}}=1$ and resistance of resonator, $\mathrm{R}=1$. Hence, the S-parameter of (5) becomes

$$
S_{21}=\frac{\left(1-1^{2}\right) 1}{(1+1)(1+1)}=0
$$

or in decibel

$$
\left|S_{21}\right|^{2} \mathrm{~dB}=20 \log _{10}(0)=\infty \mathrm{dB}
$$

where an infinite attenuation response was obtained in the matched lossy resonator. The detailed analyses of the attenuation response of the matched lossy resonators (parallel coupled line and ring resonator) are discussed in the next sections. Since there was no synthesis technique to obtain attenuation response based on the matches lossy resonator structures [9], [4], the parametric study technique was used for the attenuation response analysis. The ADS software was used for the parametric study by designing the matched lossy resonators in the schematic design tool.

\section{RESULTS AND ANALYSIS}

\subsection{Parallel Coupled Line Resonator}

For better understanding of the characteristics of matched lossy resonator using parallel coupled line structure, the analysis was begun with a conventional single resonator. The basic structure was reported in [24] for bandstop filter design, which is also known as L-shape resonator. It was coupled to the main microstrip line both electrically and magnetically.

As depicted in Figure 2(a), the single parallel coupled line resonator structure was coupled in paralleled to the microstrip transmission line. The length of the parallel coupled line resonator was half wavelength, $\lambda / 2$ at resonant frequency. The simulated frequency response $\left(S_{21}\right.$ and $\left.S_{11}\right)$ is plotted in Figure 2(b), from which the desired resonator structure parameters were determined; resonator length, $l=21.3 \mathrm{~mm}$ (resonated at $3.5 \mathrm{GHz}$ ), coupling spacing, $S=0.5 \mathrm{~mm}$, and width of microstrip line, $W=2.9 \mathrm{~mm}$.

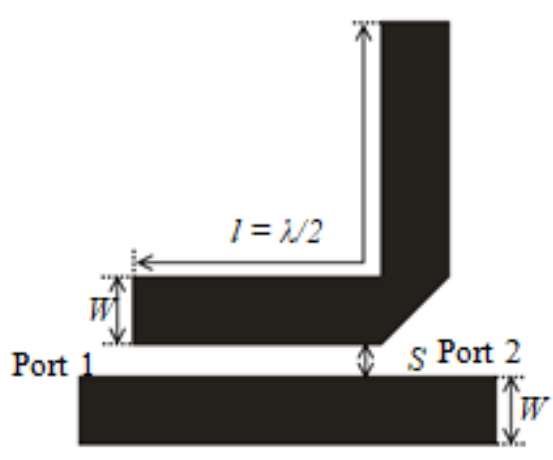

(a)

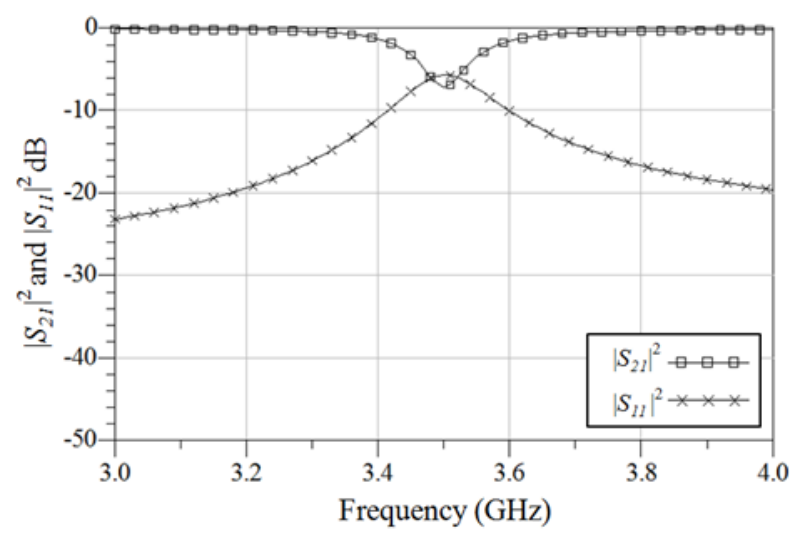

(b)

Figure 2. (a) A single parallel coupled line resonator, (b) the simulated frequency response 
It was observed that the simulated attenuation or notch of the resonator in Figure 2(b) was low, $-7 \mathrm{~dB}$ at $3.5 \mathrm{GHz}$. The simulated return loss was $-5.7 \mathrm{~dB}$. This was due to the lossy type of resonator using microstrip transmission line. From the simulated frequency response, the 3-dB bandwidth was $175 \mathrm{MHz}$. Thus, the calculated unloaded Q factor was 40.

The notch of the parallel coupled line resonator was increased by cascading more than one resonator. As depicted in Figure 3(a), double parallel coupled line resonator structures were cascaded in series with microstrip transmission line. The parallel coupled line resonators were separated with k-inverter that had been realized using quarter wavelength, $\lambda / 4$ at resonant frequency.

The simulated frequency response $\left(S_{21}\right.$ and $\left.S_{11}\right)$ was plotted in Figure 3(b), from which the desired resonator structure parameters were determined; resonator length, $l_{l}=21.3 \mathrm{~mm}$ (resonated at $3.5 \mathrm{GHz}$ ), $k_{3}$ length, $l_{2}=11 \mathrm{~mm}$ (resonated at $3.5 \mathrm{GHz}$ ), coupling spacing, $S=0.5 \mathrm{~mm}$, and width of microstrip line, $W=$ $2.9 \mathrm{~mm}$. As shown in Figure 3(b), the simulated notch of the cascaded two parallel coupled line resonator increased to $-16.8 \mathrm{~dB}$, while the return loss was $-7.2 \mathrm{~dB}$. From the simulated frequency response, the 3-dB bandwidth was $166 \mathrm{MHz}$. Thus, the calculated unloaded Q factor was 42.

Furthermore, based on the conventional parallel coupled line resonator structures that have been discussed in the previous paragraphs, notch or attenuation of the resonator could be enhanced by using matching lossy bandstop filter design proposed by [4]. Figure 4 is the desired dimension of matched parallel coupled line resonator operated at $3.5 \mathrm{GHz}$, where the resonator length, $l_{l}=21.3 \mathrm{~mm}$, length of k-inverter, $l_{2}$ $=15.4 \mathrm{~mm}$ (including microstrip bends), and width of microstrip line, $W=2.9 \mathrm{~mm}$. The length of k-inverter, $l_{2}$ is the $k_{3}$ shown in generalized model of matched lossy resonator.

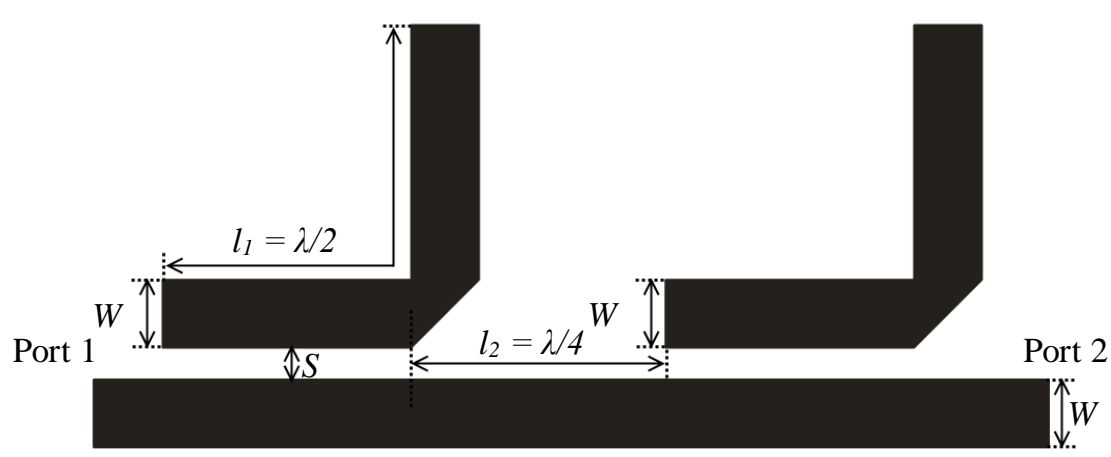

(a)

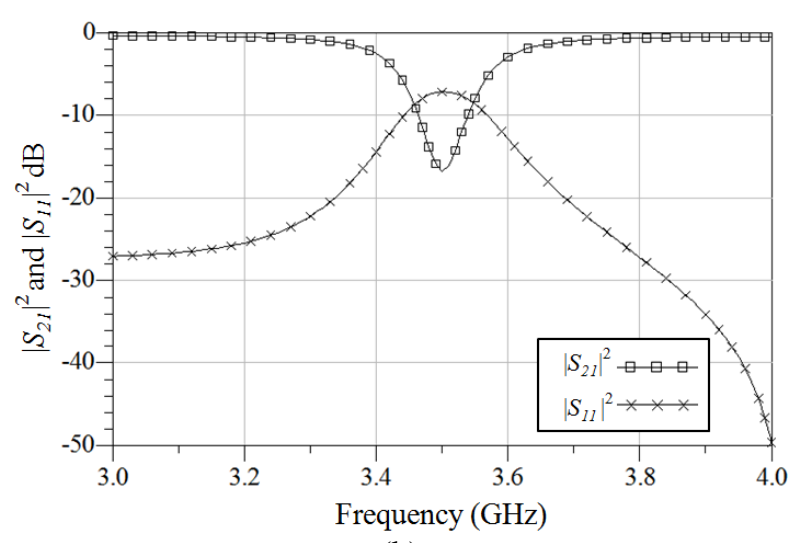

(b)

Figure 3. (a) A double parallel coupled line resonator, (b) the simulated frequency response

The parametric studies of the simulated frequency response $\left(S_{21}\right.$ and $\left.S_{11}\right)$ were plotted in Figures 5 and 6 by varying the coupling spacing, $S_{1}$ and $S_{2}$ respectively in order to determine the best high notch $\left(S_{21}\right)$ and matched return loss response $\left(S_{11}\right)$. Take note that, according to generalized model of matched lossy resonator, the $\mathrm{k}_{1}$ was realized by $S_{1}$, while the $\mathrm{k}_{2}$ was realized by $S_{2}$. From literature, perfectly matched and very high notch response could be obtained with the proper selection of values of $\mathrm{k}_{1}$ and $\mathrm{k}_{2}$. 


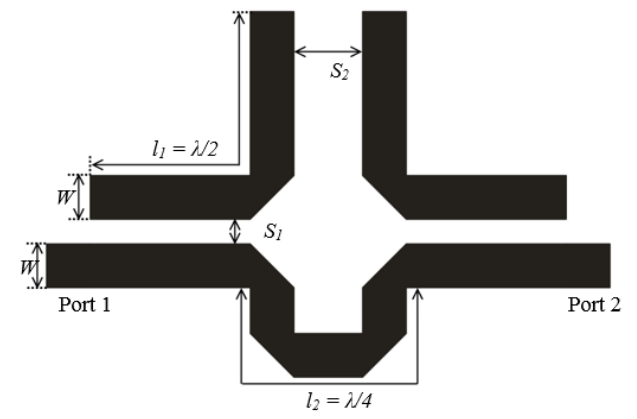

Figure 4. Matched lossy resonator using parallel coupled line and its parameters

Based on Figures 5(a) and (b), the parametric studies of $S_{21}$ and $S_{11}$ were performed in the ADS software by varying $S_{1}$ without coupling spacing between resonators, $S_{2}$. The value of $S_{1}$ was varied from 0.1 $\mathrm{mm}$ to $2.5 \mathrm{~mm}$. In this case, the circuit model in the ADS software was set without coupling of $S_{2}$ using a normal microstrip transmission line. The result showed that high notch was obtained by reducing the coupling spacing, $S_{l}$, when the electrical and magnetic field was increased. However, Figure 5(b) shows a highly unmatched response of return loss when $S_{l}$ was reduced. The matched return loss response could only be obtained by increasing the coupling spacing, $S_{l}$. Thus, there was a trade-off between high notch and matched return loss response when choosing the best $S_{l}$ value in parallel coupled line resonator design.

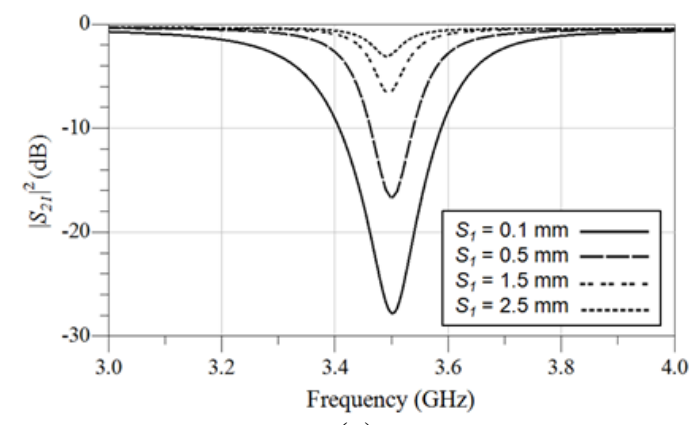

(a)

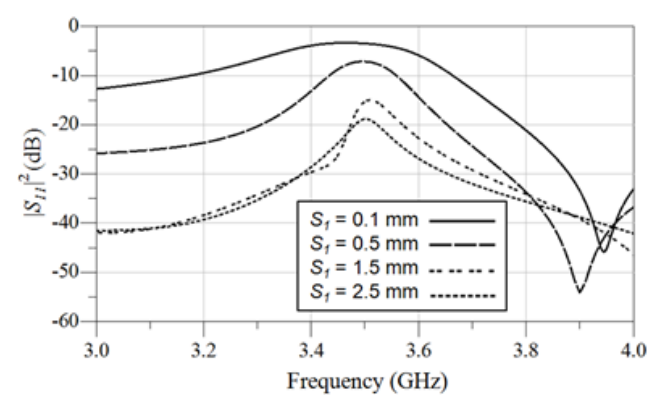

(b)

Figure 5. Parametric study of (a) S21 and (b) S11 with variation in S1 without S2

Meanwhile, in Figure 6(a) and Figure 6(b), parametric studies of $S_{21}$ and $S_{11}$ were performed by varying $S_{2}$ with fixed coupling spacing, $S_{1}=0.5 \mathrm{~mm}$. The value of $S_{2}$ was varied from $1 \mathrm{~mm}$ to $16 \mathrm{~mm}$. In this case, the circuit model in the ADS software had included the $S_{l}$ as the resonators must be coupled to the main microstrip transmission line.

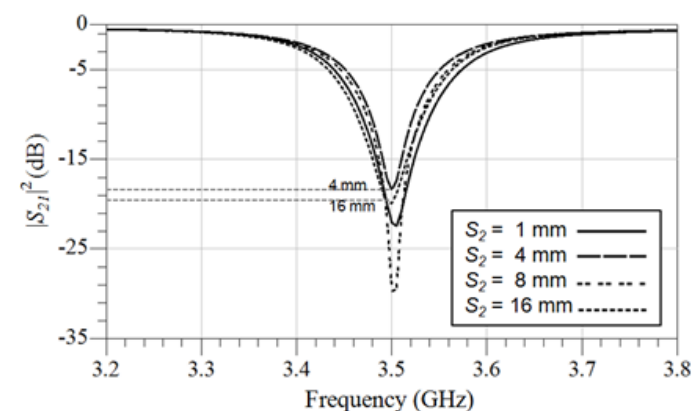

(a)

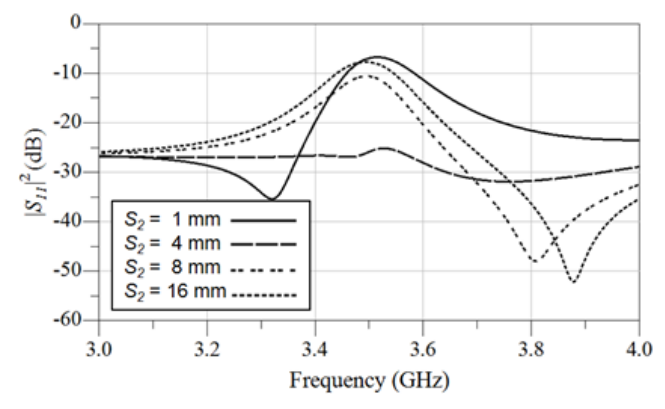

(b)

Figure 6. Parametric studies of (a) S21 and (b) S11 with variation in S2 with $\mathrm{S} 1=0.5 \mathrm{~mm}$ 
From the simulated result, it was found that a high notch with an acceptable matched return loss response was obtained at a certain value of $S_{2}$. In this parametric study, $S_{2}=8 \mathrm{~mm}$ gave the highest attenuation, $-29.8 \mathrm{~dB}$ (in Figure 6(a)) with an acceptable matched response of the return loss, -10.6 dB (in Figure 6(b)). From the simulated frequency response (for $S_{2}=8 \mathrm{~mm}$ ), the $3-\mathrm{dB}$ bandwidth was $137 \mathrm{MHz}$, giving the calculated unloaded $Q$ factor was 51 . The best matched return loss response was obtained when $S_{2}=4 \mathrm{~mm}$, which was $-25.9 \mathrm{~dB}$ return loss, but the notch response $\left(S_{21}\right)$ had slightly dropped to $-18.5 \mathrm{~dB}$. Thus, there is a trade-off between high notch and matched return loss response when choosing the best $S_{2}$ value in parallel coupled line resonator design. Therefore, based on these two simulated results in Figures 5 and 6, it was found that with careful design and proper circuit parametric study on the coupling spacing of $S_{1}$ and $S_{2}$, very high notch and matched return loss response were obtained.

\subsection{Ring Resonator}

As depicted in Figure 7, the matched lossy resonator using ring structure was side coupled to the microstrip transmission line. The total length of the matched ring resonator was full wavelength, $\lambda$, at resonant frequency. That means, for each length of $l_{l}$, the wavelength was $\lambda / 4$, and it formed a square shape of ring resonator. Furthermore, according to [25], a ring resonator with side coupled to a microstrip line produces two closely spaced, but distinct resonant frequencies were identified due to the even mode and odd mode coupling. The perturbing stub, $l_{3}$, was employed at $135^{\circ}$ of electrical length from input or output feed lines (Port 1 or Port 2) in order to gain flexibility of controlling the resonant frequency of odd mode coupling.

Besides, it has been known that the ring resonator is a dual mode resonator [3]. Thus, in order to observe the dual mode response of ring resonator in this research work, the ring structure in Figure 7 was simulated in the ADS software without perturbing stub, $l_{3}=0 \mathrm{~mm}$, and all the width of resonator and transmission line shared the same width, $W_{l}=W_{2}=2.9 \mathrm{~mm}$. The coupling spacing, $S$ was fixed at $0.5 \mathrm{~mm}, l_{l}$ $=9 \mathrm{~mm}$ and $l_{2}=10 \mathrm{~mm}$ (resonated at $3.5 \mathrm{GHz}$ ). In addition, according to [3], the asymmetrical feed lines (or side coupling) are used to the ring resonator so that two degenerate modes or splitting resonant frequencies can be exited in the ring resonator.

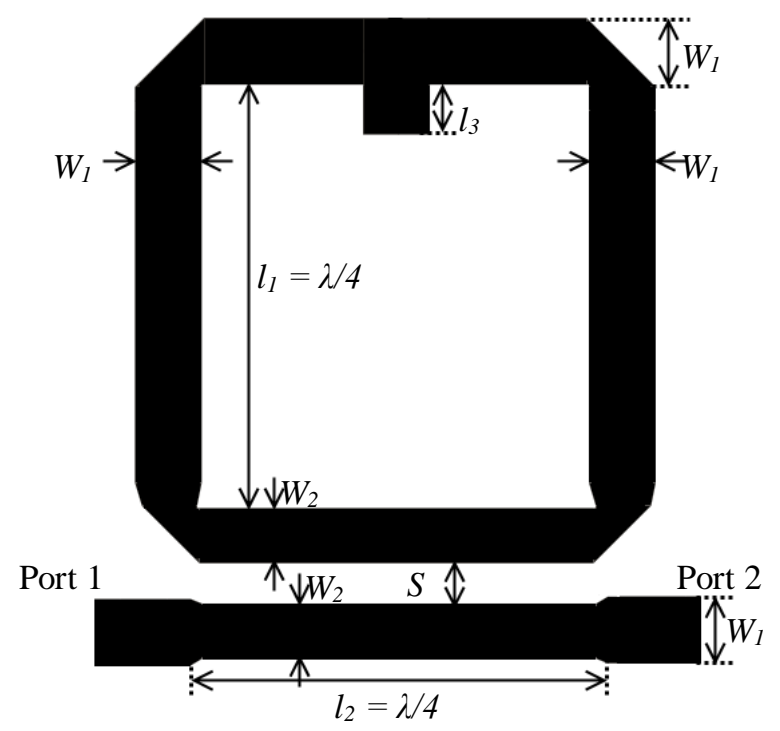

Figure 7. Matched lossy resonator using ring structure and its parameters

Thus, the simulated frequency response $\left(S_{21}\right.$ and $\left.S_{11}\right)$ in Figure 8 shows two degenerate modes or splitting resonant frequencies at $3.59 \mathrm{GHz}$ and $3.41 \mathrm{GHz}$. The simulated notches or attenuations were $-10.6 \mathrm{~dB}$ (at $3.59 \mathrm{GHz}$ ) and $-7.9 \mathrm{~dB}$ (at $3.41 \mathrm{GHz}$ ), while the simulated return loss of the two resonant frequencies were $-3.9 \mathrm{~dB}($ at $3.59 \mathrm{GHz})$ and $-5.6 \mathrm{~dB}($ at $3.41 \mathrm{GHz})$.

According to [25], the two distinct resonant peaks were identified as the even-mode (magnetic field) and odd-mode (electric field) coupling of the ring resonator to microstrip line. Hence, as depicted in Figure 8, the resonant frequency of $3.59 \mathrm{GHz}$ was due to even mode coupling, while the resonant frequency of $3.41 \mathrm{GHz}$ was due to odd mode coupling. As reported in [26], the even mode and odd mode couplings would take place if the induced magnetic or electric field is maximum at the resonator near the microstrip transmission line,

Indonesian J Elec Eng \& Comp Sci, Vol. 14, No. 1, April 2019 : 29 - 37 
inducing the corresponding electric and magnetic fields in the ring resonator and resulting in the ring resonator two simultaneous field distributions with different coupling mechanisms.

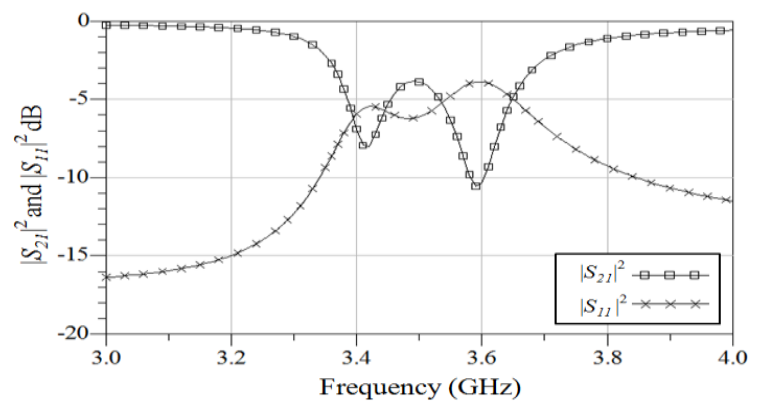

Figure 8. Simulated frequency response of dual mode ring resonator

The notch of ring resonator as shown in Figure 8 was enhanced by using matched lossy bandstop filter design proposed by Guyette [4]. In this structure, the length of $k$-inverter coupling, $l_{2}$ is the $k_{3}$, as shown in the generalized model of matched lossy resonator. Moreover, the perfectly matched and very high notch response of the ring resonator could be obtained with a proper selection of value of $k_{l}$ and $k_{2}$, whereby the value of $k_{l}$ is controlled by $S$ and $W_{2}$, while the value of $k_{2}$ is controlled by $l_{3}$. The next paragraphs explain the parametric study results of matched and analysis of notch response by varying coupling $S, W_{2}$, and $l_{3}$.

In Figure 9(a), the parametric studies of $S_{21}$ and $S_{11}$ were performed by varying $S$ without perturbed stub, $l_{3}=0 \mathrm{~mm}$ and with fixed value of width, $W_{l}=W_{2}=2.9 \mathrm{~mm}$. The value of $S$ was varied from $0.1 \mathrm{~mm}$ to 2 $\mathrm{mm}$. In this case, the circuit model in ADS software was set without perturbed stub, $l_{3}$. The result showed that even mode and odd mode couplings became weaker as coupling spacing became larger. Besides, the resonant frequencies of both even and odd modes shifted to the center frequency of ring resonator at $3.5 \mathrm{GHz}$. Meanwhile, Figure 9(b) shows a matched response of return loss when $S$ value was increased. The $S_{11}$ began to have a matched return loss response when $S>1 \mathrm{~mm}$, thus a trade-off was discovered between high notch and matched return loss response when choosing the best value for coupling spacing, $S$, in the ring resonator design.

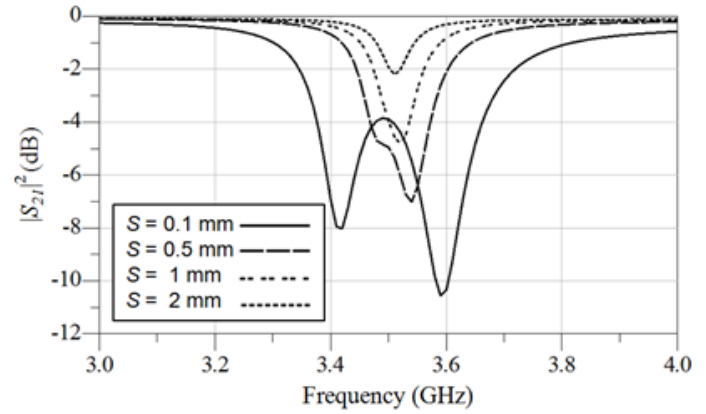

(a)

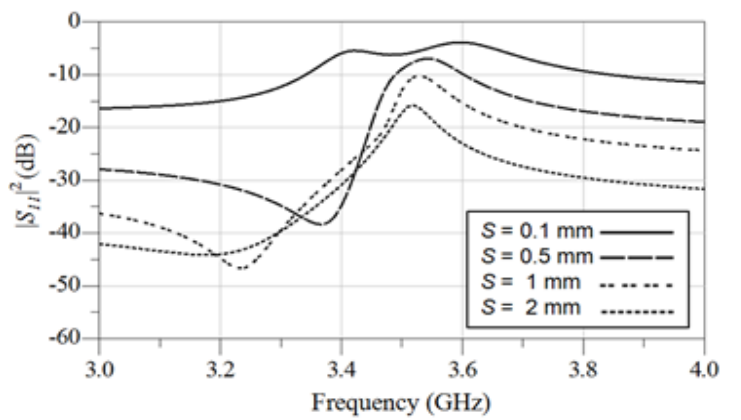

(b)

Figure 9. Parametric studies of (a) S21 and (b) S11 with variation in $\mathrm{S}$ with $13=0 \mathrm{~mm}$ and $\mathrm{W} 1=\mathrm{W} 2=$ $2.9 \mathrm{~mm}$

In Figure 10, parametric studies of $S_{21}$ and $S_{11}$ were performed by varying $l_{3}$ with fixed value of width, $W_{l}=W_{2}=2.9 \mathrm{~mm}$ and coupling spacing, $S=0.1 \mathrm{~mm}$. The value of $l_{3}$ was varied from $1 \mathrm{~mm}$ to $4 \mathrm{~mm}$. As stated earlier in this section, to control the resonant frequency of odd mode, the perturbing stub, $l_{3}$, was employed at $135^{\circ}$ of electrical length from input or output feed lines (Port 1 or Port 2). 


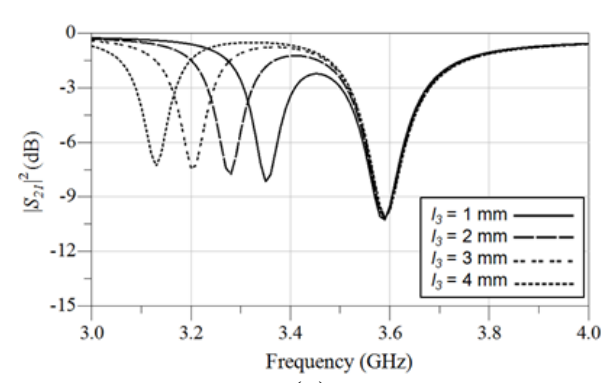

(a)

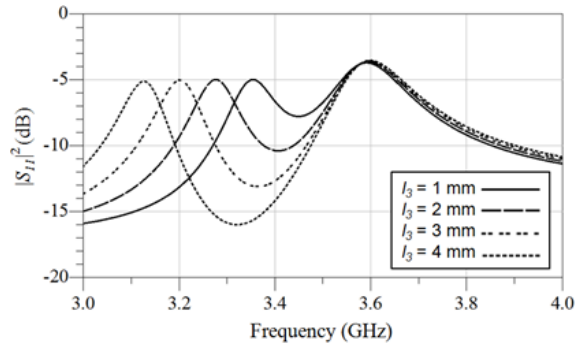

(b)

Figure 10. Parametric studies of (a) $S_{2 l}$ and (b) $S_{11}$ with variation in $l_{3}$ with $S=0.1 \mathrm{~mm}$ and $W_{l}=W_{2}=2.9$ $\mathrm{mm}$

Figures 10(a) and (b) show that the resonant frequency of odd coupling was shifted to lower frequency by increasing the length of the perturbed stub. Besides, the values of notch, $S_{21}$, and return loss, $S_{11}$, had been almost similar when the resonant frequency was shifted to lower frequencies.

In Figure 11, the parametric studies of $S_{21}$ and $S_{11}$ were performed by varying $W_{2}$ without perturbed stub, $l_{3}=0 \mathrm{~mm}$ and with fixed coupling spacing, $S=0.1 \mathrm{~mm}$. In this simulation, the value of $W_{2}$ was varied from $2.9 \mathrm{~mm}$ to $2 \mathrm{~mm}$. It was observed that the resonant frequencies of both even and odd modes were shifted to the center frequency of ring resonator at $3.5 \mathrm{GHz}$ by reducing $W_{2}$.

In Figure 11(a), the highest notch was obtained when $W_{2}=2.5 \mathrm{~mm}$, but by reducing the width lower than $2.5 \mathrm{~mm}$, it lowered the notch response. This was due to the increase in coupling spacing, $S$, simultaneously with reduction of $W_{2}$, hence weakening the even mode and odd mode couplings. On the other hand, Figure 11(b) shows a matched response of return loss when the value of $W_{2}$ was increased. The return loss, $S_{11}$, began to show matched return loss response when $W_{2}<2 \mathrm{~mm}$, thus a trade-off between high notch and matched return loss response was found when choosing the best coupling spacing, $W_{2}$, in the ring resonator design.

Based on the simulated results in Figures 9, 10, and 11, it was found that a very high notch and matched return loss response were obtained with careful design and proper circuit parametric study on the coupling spacing, $S$, width at coupling lines, $W_{2}$, and perturbed stub length, $l_{3}$.

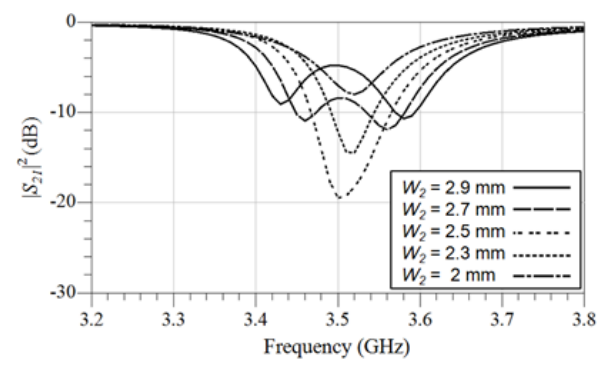

(a)

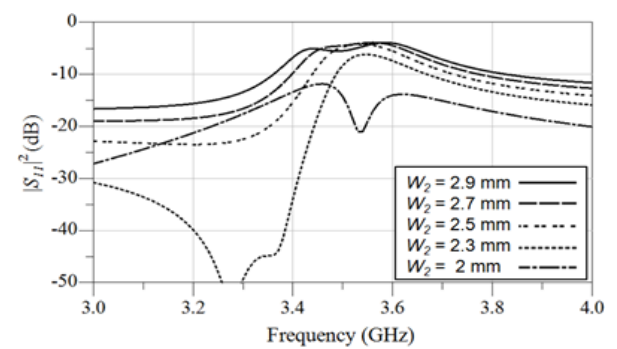

(b)

Figure 11. Parametric studies of (a) $S_{21}$ and (b) $S_{11}$ with variation in $W_{2}$ with $l_{3}=0 \mathrm{~mm}$ and $S=0.1 \mathrm{~mm}$

\section{CONCLUSION}

Parametric studies of dual mode parallel coupled line and ring resonators for matched bandstop filter design was discussed. For parallel coupled line, based on the two simulated results in Figures 5 and 6, it was found that with careful design and proper circuit parametric study on the coupling spacing of $S_{1}$ and $S_{2}$, very high notch and matched return loss response were obtained. In contrast, based on the simulated results in Figures 9, 10, and 11, it was found that a very high notch and matched return loss response were obtained with careful design and proper circuit parametric study on the coupling spacing, $S$, width at coupling lines, $W_{2}$, and perturbed stub length, $l_{3}$. Therefore, the crucial isuue in the parallel coupled line and ring resonators is the variation or tolerance of coupling gap. The smallest changes of coupling gap could significantly change the response. 


\section{ACKNOWLEDGEMENTS}

Authors would like to greatly express their thanks and appreciation to Universiti Teknikal Malaysia Melaka UTeM Zamalah Scheme for their encouragement, help and financially supporting to complete this research work. The publication of this paper was funded by the Short Term Research Grant under the research grant No. PJP/2018/FTK(A1)/S01617.

\section{REFERENCES}

[1] U. Rafique, S. A. Ali, M. T. Afzal, and M. Abdin, "Bandstop Filter Design for GSM Shielding Using Frequency Selective Surfaces," vol. 2, no. 6, pp. 846-850, 2012.

[2] B. Nasiri, A. Errkik, J. Zbitou, A. Tajmouati, L. El Abdellaoui, and M. Latrach, "A New Compact and Wide-band Band-stop Filter Using Rectangular SRR,” vol. 16, no. 1, pp. 110-117, 2018.

[3] K. Chang and L.-H. Hsieh, Microwave Ring Circuits and Related Structures, 2nd ed. Wiley-Interscience, 2004.

[4] A. C. Guyette, et al., "Perfectly-Matched Bandstop Filters using Lossy Resonators," in 2005 IEEE MTT-S International Microwave Symposium Digest, 2005, pp. 517-520.

[5] A. Guyette, "Controlled Agility: Frequency-Agile Planar Filters with Advanced Features," IEEE Microw. Mag., no. July, pp. 33-42, 2014.

[6] D. R. Jachowski and A. C. Guyette, "Sub-Octave-Tunable Microstrip Notch Filter," in Microwave Symposium Digest, 2009. MTT '09. IEEE MTT-S International, 2009, pp. 99-102.

[7] B. A. Adoum and P. W. Wong, "Miniaturized Matched Band-Stop Filter Based Dual Mode Resonator," in National Postgraduate Conference (NPC), 2011, pp. 7-9.

[8] A. C. Guyette, "Intrinsically Switched Varactor-Tuned Filters and Filter Banks," IEEE Trans. Microw. Theory Tech., vol. 60 , no. 4, pp. 1044-1056, 2012.

[9] A. C. Guyette, I. C. Hunter, and R. D. Pollard, "Design of Absorptive Microwave Filters using Allpass Networks in a Parallel-Cascade Configuration," in Microwave Symposium Digest, 2009. MTT '09. IEEE MTT-S International, 2009, pp. 733-736.

[10] P. W. Wong, I. C. Hunter, and R. D. Pollard, "Matched Bandstop Resonator with Tunable K-Inverter," in 2007 European Microwave Conference, 2007, pp. 664-667.

[11] R. Alahnomi, N. Binti, A. Hamid, Z. Zakaria, T. Sutikno, and A. Azuan, "Microwave Planar Sensor for Permittivity Determination of Dielectric Materials," vol. 11, no. 1, pp. 362-371, 2018.

[12] N. A. Wahab et al., "Bandpass filter Based on Ring Resonator at RF Frequency above 20 GHz," vol. 9, no. 3, pp. 680-684, 2018.

[13] D. R. Jachowski, “Tunable Lumped-Element Notch Filter with Constant Bandwidth," in Wireless Information Technology and Systems (ICWITS), 2010 IEEE International Conference on, 2010, vol. 3, no. 1, pp. 1-4.

[14] J. Lee, T. C. Lee, and W. J. Chappell, "Lumped-Element Realization of Absorptive Bandstop Filter With Anomalously High Spectral Isolation,” IEEE Trans. Microw. Theory Tech., vol. 60, no. 8, pp. 2424-2430, 2012.

[15] M. K. Zahari, B. H. Ahmad, N. A. Shairi, and P. W. Wong, "Reconfigurable Dual-Mode Ring Resonator Matched Bandstop Filter," in 2012 IEEE Symposium on Wireless Technology and Applications (ISWTA), 2012, pp. 71-74.

[16] M. K. Zahari, B. H. Ahmad, N. A. Shairi, and P. W. Wong, "Reconfigurable Matched Bandstop Filter," in 2011 IEEE International RF and Microwave Conference (RFM 2011), 2011, pp. 230-233.

[17] M. K. Zahari, B. H. Ahmad, W. P. Wen, and N. A. Shairi, "Switchable Absorptive Bandstop to Bandpass Filter Using Dual-Mode Ring Resonator," in 2016 IEEE Asia-Pacific Conference on Applied Electromagnetics (APACE), 2016, pp. 248-251.

[18] M. K. Zahari, B. H. Ahmad, P. W. Wong, and N. A. Shairi, "Switchable Bandstop to Bandpass Filter Using ParallelCoupled Resonator," in Microwave Conference (APMC), 2017 IEEE Asia Pacific, 2017, pp. 5-8.

[19] N. A. Shairi, B. H. Ahmad, and P. W. Wong, "Absorptive SPDT Switch Design using Cascaded Switchable ParallelCoupled Stub Resonator and Switchable Radial Stub Resonator," Reg. 10 Symp. 2014 IEEE, vol. 2, pp. 310-314, 2014.

[20] N. A. Shairi, B. H. Ahmad, M. K. Zahari, and P. W. Wong, "The Potential Ap pplication of Switchabl le Matched Parallel-Coupled Stub Resonator in SPD DT Discrete Switch Design," in 2012 IEEE Asia-Pacific Conference on Applied Electromagnetics (APACE 2012), 2012, pp. 372-376.

[21] N. A. Shairi, P. W. Wong, and B. H. Ahmad, "Switchable matched ring resonator in SPDT discrete switch design for WiMAX and LTE in $3.5 \mathrm{GHz}$ band," in Asia-Pacific Microwave Conference 2014, 2014, vol. 7, pp. 759-761.

[22] N. A. Shairi, A. M. Zobilah, B. H. Ahmad, and Z. Zakaria, "Design Comparison of RF SPDT Switch with Switchable Resonators for WiMAX and LTE in 3 . 5 GHz Band," vol. 12, no. 20, pp. 9614-9618, 2017.

[23] M. Y. Algumaei, N. A. Shairi, Z. Zakaria, A. M. S. Zobilah, and B. H. Ahmad, "Analysis of Open Stub Resonator and its Application in Dual Isolation Band of SPDT Switch Design,” vol. 6, no. 6, pp. 2900-2910, 2016.

[24] J. Hong and M. J. Lancaster, Microstrip Filters for RF/Microwave Applications. New Jersey: John Wiley \& Sons, Inc., 2001.

[25] S. L. Lu and A. M. Ferendeci, "Coupling modes of a ring resonator side coupled to a microstrip line," Electron. Lett., vol. 30, no. 16, pp. 1314-1315, 1994.

[26] S.-L. Lu and M. F. Altan, "Coupling Parameters for a Side-Coupled Ring Resonator and a Microstrip Line," Microw. Theory Tech. IEEE Trans., vol. 44, no. 6, pp. 953-956, 1996. 